\title{
On Proportional Fair Scheduling in Multi-Antenna Wireless Mesh Networks-Theoretical Analysis
}

\author{
Erwu Liu and Kin K. Leung \\ Department of Electrical and Electronic Engineering \\ Imperial College \\ London, United Kingdom \\ \{erwu.liu, kin.leung@imperial.ac.uk\}
}

\begin{abstract}
Proportional fair scheduling (PFS) provides good balance between throughput and fairness via multi-user diversity and gametheoretic equilibrium. Very little analytical work exists on understanding the performance of PFS. Moreover, most researches on PFS are for cellular networks and typically use linear rate model or logarithm rate model to simplify the theoretical analysis of PFS. Since the linear rate model only applies to very small SINR, most researchers prefer the logarithm rate model in their study on PFS. While previous work which is based on the logarithm rate model provides good estimate of the PFS throughput in Rayleigh fading single-antenna cellular networks, they are not valid for multi-antenna wireless mesh networks. In this paper, PFS in multi-antenna wireless mesh networks under Rayleigh fading is discussed. Specifically, we assume that orthogonal frequency-division multiplexing (OFDM) and single-input multi-output (SIMO) techniques are used in the network. In addition, a new mathematical analysis of PFS that applies to both Rayleigh and Rician fading is presented. Simulations are conducted to verify our analytic results on PFS in the proposed mesh network. To the best of our knowledge, this work is the first one investigating the PFS problem in multi-antenna mesh networks.
\end{abstract}

Keywords- proportional fair scheduling, Rayleigh fading, orthogonal frequency-division multiplexing, single-input multiple-output

\section{INTRODUCTION}

Since its first presence [1], the PFS algorithm has attracted lots of attention and currently implemented in $3 \mathrm{G}$ wireless networks [2]. Until now, one can only see very limited analysis results on PFS [3-7], whose derivations typically assume a linear rate model or logarithm rate model and/or use a simplified form of the original PFS preference metric. In the linear rate model, the instantaneous data rate $R$ of a user is proportional to the received signal to interference-plus-noise ratio (SINR), while in the logarithm rate model there is a logarithmic relationship between the received SINR and the feasible data rate. While most researchers prefer the logarithm rate model to the linear one in the studies of PFS, to simplify the problem, earlier researches $[3,5,6]$ typically use the linear rate model. As stated in [3] however, the linear rate model is usually a reasonable approximation for small SINR and is not accurate when multiple modulations or codings are used. Recently, some theoretical results under the logarithm rate model have been presented [7]. These results provide valuable guideline on the system design of single-antenna-i.e., singleinput-single-output (SISO)_PFS-enabled cellular networks.

With the increasing tendency of implementing multiantenna technique in wireless mesh networks, it is interesting to evaluate the PFS performance in multi-antenna-i.e., SIMO, multiple-input-single-output (MISO) or multiple-input- multiple-output (MIMO)—wireless mesh networks. In this paper, we propose a full duplex distributed PFS-enabled multiple-antenna mesh (DPMAM) network. Specifically, we assume each node has multiple transmit and receive antennas, also OFDM and SIMO techniques are used in the full duplex mesh network. Our simulations have found that existing analytic results which are based on the logarithm rate model are not valid in multi-antenna wireless mesh environments. For this reason, more appropriated rate model other than the logarithm one should be used. A new mathematical framework for the PFS research is then developed in the paper. In fact, $[8,9]$ point out that, for a $t \times r$ MIMO link over Rayleigh fading channel, where $t$ and $r$ are the numbers of transmit and receive antennas, the feasible data rate over a Rayleigh fading environment can be accurately modeled by a Gaussian distribution with the mean and variance (in bps/Hz) of the MIMO link given by

$$
\begin{gathered}
E[R]=\int_{0}^{\infty} \omega(\lambda) \sum_{k=0}^{m-1} \frac{k ! \lambda^{n-m} e^{-\lambda}}{(k+n-m) !}\left[L_{k}^{n-m}(\lambda)\right]^{2} d \lambda \\
\sigma_{R}^{2}=m \int_{0}^{\infty} \omega^{2}(\lambda) p(\lambda) d \lambda-\sum_{i=0}^{m-1} \sum_{j=0}^{m-1}\left[\frac{i ! j !}{(i+n-m) !(j+n-m) !}\right] \\
\quad \times\left(\int_{0}^{\infty} \lambda^{n-m} e^{-\lambda} L_{i}^{n-m}(\lambda) L_{j}^{n-m}(\lambda) \omega(\lambda) d \lambda\right)^{2}
\end{gathered}
$$

where $m=\min (t, r), n=\max (t, r), L_{k}^{n-m}($.$) is generalized Laguerre$ polynomials of order $k, p(\lambda)=\frac{1}{m} \sum_{i=0}^{m-1} \frac{i ! \lambda^{n-m} e^{-\lambda}}{(i+n-m) !}\left[L_{i}^{n-m}(\lambda)\right]^{2}$ is the $p d f$ of the nonnegative eigenvalues of the channel matrix, $\overline{\operatorname{SINR}}$ is the average SINR, and $\omega(\lambda)=\log _{2}(1+\overline{\operatorname{SINR}} \times \lambda / t)$.

The rest of the paper is structured as follows. A distributed PFS-enabled multi-antenna mesh (DPMAM) network is proposed in Section II. After that, Section III first conducts theoretical analysis of the PFS throughput with the assumption that the feasible rate can be approximated by a Gaussian distribution, and then extends the analytic results to the proposed DPMAM network. In Section IV, we present simulation results to verify the theoretical findings, followed by the conclusion and future work in Section V.

\section{Distributed PFS-EnABLEd Multi-Antenna MeSH}

Refer to Figure 1 for a wireless mesh network. $N$ nodes (denoted as nodes $n_{1}, n_{2}, \ldots, n_{N}$ ) are placed within the network. 


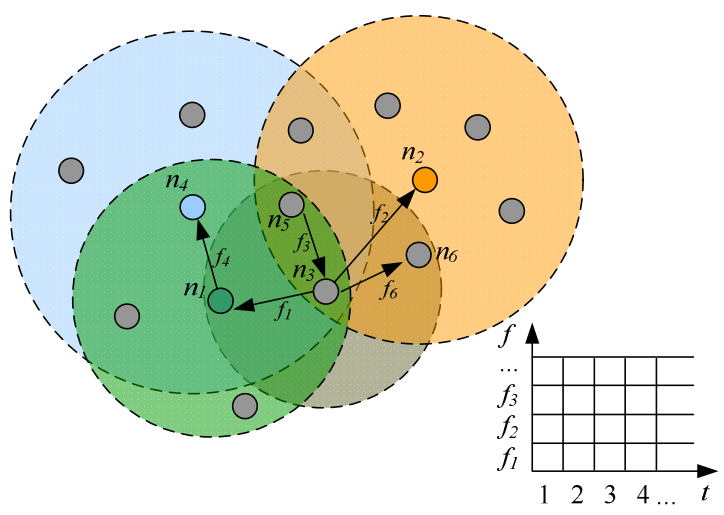

Figure 1. OFDM full duplex mesh network

We take following assumptions:

- Each node is equipped with multiple receive antennas and multiple transmit antennas.

- OFDM is used to allocate resources in frequency and time dimensions, i.e., the whole bandwidth is divided into hundreds of subcarriers, which are then bunched into subchannels $f_{j}, j=1,2, \ldots N$, and time is divided into small scheduling slots

- $n_{j}$ uses subchannel $f_{j}$ to receive from its neighbors. All the neighbors of $n_{j}$ use $f_{j}$ to transmit to $n_{j}$.

- When $n_{i}$ is scheduled by $n_{j}$ for transmission, $n_{i}$ uses SIMO to transmit on its $j^{\text {th }}$ transmit antennas to $n_{j}$. Moreover, to avoid the embarrassment where there isn't enough transmit antennas that a node can use if it is scheduled by multiple neighbors simultaneously, each node is equipped with enough transmit antennas that is at least the number of neighbors it can transmit to.

With these assumptions, each node can PF schedule its neighbors independently and full duplex transmission can be used while at the same time the interference from other nodes is greatly reduced. Correspondingly, the wireless mesh network shown in Figure 1 is called distributed PFS-enabled multi-antenna mesh (DPMAM) network. Refer to Figure 1, in a time slot $k, n_{l}$ PF schedules its neighboring node $n_{3}$ to transmit to $n_{l}$ using subchannel $f_{l}$ in next slot $k+1$. In the same slot, $n_{4} \mathrm{PF}$ schedules $n_{1}$ to transmit to $n_{4}$ using subchannel $f_{4}$. $n_{2} \mathrm{PF}$ schedules $n_{3}$ to transmit to $n_{2}$ using subchannel $f_{2}$. $n_{3} \mathrm{PF}$ schedules $n_{5}$ to transmit to $n_{3}$ using subchannel $f_{3}$ and $n_{6} \mathrm{PF}$ schedules $n_{3}$ to transmit to $n_{6}$ using subchannel $f_{6}$. Obviously, $n_{l}$ and $n_{3}$ have duplex transmission in next slot.

For a DPMAM network, the system performance will be significantly improved due to three facts:

a) Multiple antennas increase throughput;

b) The interference from other nodes are negligible since the subchannels are orthogonal each other, so full duplex transmission can be used;

c) The PFS algorithm improves performance by using multiuser diversity, and one will expect more PFS benefit as there is more diversity in a DPMAM network than a traditional cellular network.
We now conduct analysis for DPMAM networks.

\section{PFS IN DPMAM NETWORK: THEORETICAL ANALYSIS}

Since each node in a DPMAM network $P F$ schedules its neighbor independently, node $n_{j}$ can be considered as the base station (BS) of a $N_{j}$-user cellular network, where $N_{j}$ is the total number of neighbors that $n_{j}$ can receive from. Now, the PFS problem in a DPMAM network reduces to the PFS problem in a cellular network. The most challenge in analyzing a DPMAM network lies in the fact that all existing PFS theoretical results from cellular networks are valid only for SISO links. [7] presents a simple extension to MIMO cases. For reference, we quote here the existing theoretical result and its extension to MIMO cellular networks [7]:

$$
\left\{\begin{array}{l}
T_{k, \text { SISO }}=W \int_{0}^{\infty} \log _{2}\left(1+\overline{\operatorname{SINR}_{k}} \times x\right) \times e^{-x} \times\left(1-e^{-x}\right)^{N-1} d x \\
(\text { SISO case, logarithm rate model }) \\
T_{k, \text { MIMO }}=W \times N_{A} \times \int_{0}^{\infty} \log _{2}\left(1+\frac{\overline{\operatorname{SINR}_{k}}}{N_{A}} \times x\right) \times e^{-x} \times\left(1-e^{-x}\right)^{N-1} d x \\
\left(t \times r \text { MIMO case with } t=r=N_{A}, \text { logarithm rate model }\right)
\end{array} .\right.
$$

where $W$ is the system bandwidth, $N$ is the total user number in the network, $\overline{\operatorname{SINR}}_{k}$ and $T_{k}$ are the average received SINR and average throughput of user $k$, respectively.

Simulations (see Figure 2) show that the MIMO extension from the existing theoretical result for SISO cellular networks provides very rough estimation of the PFS throughput in multiantenna systems. In fact, it is because the logarithm rate model is valid only for SISO links. For this reason, the Gaussian approximation (GA) method (i.e., feasible rate can be approximated by a Gaussian distribution) $[8,9]$ other than the logarithm rate model is used in our mathematical analysis.

\section{A. PFS-GA-based Analysis in Cellular Networks}

For a cellular network where $N$ mobile stations (denoted as users $n_{1}, n_{2}$, and $n_{N}$ ) are located within the cell served by a single base station $(B S)$, let's consider the problem where these $N$ users wishing to transmit data to the $B S$, and the rates of transmission are randomly varying due to channel fluctuations. Time is divided into small scheduling intervals called slots. The selection of the user to schedule is based on a balance between the current possible rates and fairness. The proportional fair scheduling (PFS) $[1,3,4,7]$ performs this by comparing the ratio of the instantaneous rate for each node to its average throughput tracked by an exponential moving average, which is defined as the preference metric. The user with the maximum preference metric will be selected for transmission at the next scheduling slot. This is described mathematically as follows. The end of slot $m$ is called time $m$. In next time slot $m+1$, the instantaneous data rate of node $j$ will be $R_{j}[m+1]$. Its $k$-point moving average throughput up to time $m$ is denoted by $\mu_{j ; k}[m]$, and the preference metric by $M_{j ; k}[m+1]=R_{j}[m+1] / \mu_{j ; k}[m]$.

User $i=\arg \max _{j} M_{j ; k}[m+1]=\arg \max _{j} R_{j}[m+1] / \mu_{j ; k}[m]$ is scheduled to transmit in next time slot $m+1$. The moving average throughput of user $j$ up to time $m+1$ is updated by 
$\mu_{j ; k}[m+1]=\left(1-\frac{1}{k}\right) \mu_{j ; k}[m]+I_{j}[m+1] \times \frac{R_{j}[m+1]}{k}$.

where $I_{j}[m+1]$ is the indicator function of the event that user $j$ is scheduled to transmit in time slot $m+1$.

$I_{j}[m+1]=\left\{\begin{array}{l}1, j \text { is scheduled to transmit in slot } m+1 . \\ 0, \text { else }\end{array}\right.$.

From (4), we have,

$E\left[\mu_{j ; k}[m+1]\right]=\left(1-\frac{1}{k}\right) E\left[\mu_{j ; k}[m]\right]+\frac{E\left[I_{j}[m+1] R_{j}[m+1]\right]}{k}$.

where $E($.$) is the statistical average.$

Assuming wide-sense stationary $\mu_{j ; k}$ and applying Bayes's theorem, we can write (6) as

$$
\begin{aligned}
E\left[\mu_{j ; k}[m]\right] & \left.=E\left|R_{j}[m+1]\right| I_{j}[m+1]=1\right] \times \operatorname{Pr}\left(I_{j}[m+1]=1\right) \\
& =\operatorname{Pr}\left(I_{j}[m+1]=1\right) \times \int_{0}^{\infty} x f_{R_{j}}\left(x \mid I_{j}[m+1]=1\right) d x \\
& =\int_{0}^{\infty} x f_{R_{j}}(x) \operatorname{Pr}\left(I_{j}[m+1]=1 \mid R_{j}[m+1]=x\right) d x
\end{aligned}
$$

where $\operatorname{Pr}\left(I_{j}[m+1]=1\right)$ is the average probability that user $j$ will be scheduled in time slot $m+1, \operatorname{Pr}\left(I_{j}[m+1]=1 \mid R_{j}[m+1]=x\right)$ is the corresponding conditional probability and $f_{R j}($.$) is the$ probability density function ( $p d f)$ of $R_{j}$.

For statistically independent $R_{j}$, we have

$$
\begin{aligned}
\operatorname{Pr}\left(I_{j}[m+1]=1 \mid R_{j}[m+1]=x\right) & =\operatorname{Pr}\left(\forall i \neq j, \frac{R_{i}[m+1]}{\mu_{i ; k}[m]}<\frac{x}{\mu_{j ; k}[m]}\right) . \\
& =\prod_{i=1, i \neq j}^{N} F_{R_{i}}\left(\frac{\mu_{i ; k}[m]}{\mu_{j ; k}[m]} x\right)
\end{aligned}
$$

We further assume $\mu_{j ; k}$ is first-order ergodic. Taking the limit on both side of (7) yields

$$
\begin{aligned}
E\left\lfloor\mu_{j}\right\rfloor & =\lim _{k \rightarrow \infty} E\left\lfloor\mu_{j ; k}[m]\right] \\
& =\int_{0}^{\infty} x f_{R_{j}}(x) \prod_{i=1, i \neq j}^{N} F_{R_{i}}\left(\lim _{k \rightarrow \infty} \frac{\mu_{i ; k}[m]}{\mu_{j ; k}[m]} x\right) d x . \\
& =\int_{0}^{\infty} x f_{R_{j}}(x) \prod_{i=1, i \neq j}^{N} F_{R_{i}}\left(\frac{E\left[\mu_{i}\right]}{E\left[\mu_{j}\right]} x\right) d x
\end{aligned}
$$

Simulations (see Figure 3 and 4) have found that, for PFS over Rayleigh fading channels, it satisfies $\sigma_{R i} / \sigma_{R j} \leq E\left[\mu_{i}\right] / E\left[\mu_{j}\right] \leq E\left[R_{i}\right] / E\left[R_{j}\right]$ given $0 \leq E\left[R_{j}\right] \leq E\left[R_{i}\right]$, where $\sigma_{R i}$ is the standard deviation of $R_{i}$. We than have

$$
\begin{aligned}
& \prod_{\forall i \neq j, E\left[R_{i}\right] \geq E\left[R_{j}\right]}^{N} F_{R_{i}}\left(\frac{\sigma_{R_{i}}}{\sigma_{R_{j}}} x\right) \times \prod_{\forall i \neq j, E\left[R_{i}\right] E E\left[R_{j}\right.}^{N} F_{R_{j}}\left(\frac{E\left[R_{i}\right]}{E\left[R_{j}\right]} x\right) \\
& \leq \prod_{i=1, i \neq j}^{N} F_{R_{i}}\left(\frac{E\left[\mu_{i}\right]}{E\left[\mu_{j}\right]} x\right) \leq \\
& \prod_{\forall i \neq j, E\left[R_{i}\right] \geq E\left[R_{j}\right]}^{N} F_{R_{i}}\left(\frac{E\left[R_{i}\right]}{E\left[R_{j}\right]} x\right) \times \prod_{\forall i \neq j, E\left[R_{i}\right] \leqslant E\left[R_{j}\right]}^{N} F_{R_{i}}\left(\frac{\sigma_{R_{i}}}{\sigma_{R_{j}}} x\right)
\end{aligned}
$$

Looser bounds can be obtained from (10),

$$
\begin{aligned}
& \prod_{\forall i \neq j, E\left[R_{i}, E E\left[R_{j}\right]\right.}^{N} F_{R_{i}}\left(\frac{\sigma_{R_{i}}}{\sigma_{R_{j}}} x\right) \times \prod_{\forall i \neq j, E\left[R_{i}\right\} E\left[R_{j}\right]}^{N} F_{R_{i}}\left(\frac{E\left[R_{i}\right]}{E\left[R_{j}\right]} x+E\left[R_{i}\right]-\frac{\sigma_{R_{i}}}{\sigma_{R_{j}}} E\left[R_{j}\right]\right) . \\
& \leq \prod_{i=1, i \neq j}^{N} F_{R_{i}}\left(\frac{E\left[\mu_{i}\right]}{E\left[\mu_{j}\right]} x\right) \leq \\
& \leq \prod_{\forall i \neq j, E\left[R_{i}, \geq E\left[R_{i}\right]\right.}^{N} F_{R_{i}}\left(\frac{E\left[R_{i}\right]}{E\left[R_{j}\right]} x+E\left[R_{i}\right]-\frac{\sigma_{R_{i}}}{\sigma_{R_{j}}} E\left[R_{j}\right]\right) \times \prod_{\forall i \neq j, E\left[R_{i} \mid k E\left[R_{j}\right]\right.}^{N} F_{R_{i}}\left(\frac{\sigma_{R_{i}}}{\sigma_{R_{j}}} x\right)
\end{aligned}
$$

It is easy to prove that

$$
\begin{aligned}
& \prod_{\forall i \neq j, E\left[R_{i}, \geq E\left[R_{j}\right]\right.}^{N} F_{R_{i}}\left(\frac{\sigma_{R_{i}}}{\sigma_{R_{j}}} x\right) \times \prod_{\forall i \neq j, E\left[R_{i}\right\} E\left[R_{i}\right]}^{N} F_{R_{j}}\left(\frac{E\left[R_{i}\right]}{E\left[R_{j}\right]} x+E\left[R_{i}\right]-\frac{\sigma_{R_{i}}}{\sigma_{R_{j}}} E\left[R_{j}\right]\right) \\
& \leq \prod_{i=1, i \neq j}^{N} F_{R_{i}}\left(\frac{\sigma_{R_{i}}}{\sigma_{R_{j}}} x+E\left[R_{i}\right]-\frac{\sigma_{R_{i}}}{\sigma_{R_{j}}} E\left[R_{j}\right]\right) \leq \\
& \leq \prod_{\forall i \neq j, E\left[R_{i}, E\left[R_{j}\right]\right.}^{N} F_{R_{i}}\left(\frac{E\left[R_{i}\right]}{E\left[R_{j}\right]} x+E\left[R_{i}\right]-\frac{\sigma_{R_{i}}}{\sigma_{R_{j}}} E\left[R_{j}\right]\right) \times \prod_{\forall i \neq j, E, E R, k E\left[R_{j}\right]}^{N} F_{R_{i}}\left(\frac{\sigma_{R_{i}}}{\sigma_{R_{j}}} x\right)
\end{aligned}
$$

Now we have the following approximation

$$
\prod_{i=1, i \neq j}^{N} F_{R_{i}}\left(\frac{E\left[\mu_{i}\right]}{E\left[\mu_{j}\right]} x\right) \approx \prod_{i=1, i \neq j}^{N} F_{R_{i}}\left(\frac{\sigma_{R_{i}}}{\sigma_{R_{j}}} x+E\left[R_{i}\right]-\frac{\sigma_{R_{i}}}{\sigma_{R_{j}}} E\left[R_{j}\right]\right) \text {. }
$$

Denote $E\left[R_{j}\right] / \sigma_{R j}$ by $M_{j}$. Substituting (13) into (9) yields

$$
\begin{aligned}
E\left[\mu_{j}\right] & =\int_{0}^{\infty} x f_{R_{j}}(x) \prod_{i=1, i \neq j}^{N} F_{R_{i}}\left(\frac{E\left[\mu_{i}\right]}{E\left[\mu_{j}\right]} x\right) d x \\
& \approx \int_{0}^{\infty} x f_{R_{j}}(x) \prod_{i=1, i \neq j}^{N} F_{R_{i}}\left(\frac{\sigma_{R_{i}}}{\sigma_{R_{j}}} x+E\left[R_{i}\right]-\frac{\sigma_{R_{i}}}{\sigma_{R_{j}}} E\left[R_{j}\right]\right) d x \\
& =\sigma_{R_{j}} \int_{-M_{j}}^{\infty}\left(y \sigma_{R_{j}}+E\left[R_{j}\right]\right) f_{R_{j}}\left(y \sigma_{R_{j}}+E\left[R_{j}\right]\right) \prod_{i=1, i \neq j}^{N} F_{R_{i}}\left(y \sigma_{R_{i}}+E\left[R_{i}\right]\right) d x
\end{aligned}
$$

As stated earlier, $R_{i}$ of a SISO/SIMO/MISO/MIMO link over Rayleigh/Rician fading channels can be accurately approximated by a Gaussian distribution [9], we can then rewrite (14) as

$$
\begin{aligned}
E\left[\mu_{j}\right] & \approx \sigma_{R_{j}} \int_{-M_{j}}^{\infty}\left(y \sigma_{R_{j}}+E\left[R_{j}\right]\right) f_{R_{j}}\left(y \sigma_{R_{j}}+E\left[R_{j}\right]\right) \prod_{i=1, i \neq j}^{N} F_{R_{i}}\left(y \sigma_{R_{i}}+E\left[R_{i}\right]\right) d x \\
= & \sigma_{R_{j}} \int_{-M_{j}}^{\infty}\left(y \sigma_{R_{j}}+E\left[R_{j}\right]\right) f_{R_{j}}\left(y \sigma_{R_{j}}+E\left[R_{j}\right]\right)\left(F_{(0,1)}(y)\right)^{N-1} d x \\
= & \frac{E\left[R_{j}\right]}{N} \times\left(1-\left[F_{(0,1)}\left(-E\left[R_{j}\right] / \sigma_{R_{j}}\right)\right]^{\mathrm{N}}\right) \\
& +\int_{-M_{j}}^{\infty} y \sigma_{R_{j}} f_{(0,1)}(y) \times\left[F_{(0,1)}(y)\right]^{N-1} d x \\
& \approx \frac{E\left[R_{j}\right]}{N}+\int_{-M_{j}}^{\infty} y \sigma_{R_{j}} f_{(0,1)}(y) \times\left[F_{(0,1)}(y)\right]^{N-1} d x
\end{aligned}
$$

(15) is our theoretical expression of the PFS throughput in both Rayleigh and Rician fading cellular networks. It has a very clear physical meaning: the first item in the $R H S$ represents the average throughput from round-robin $(R R)$ scheduling while the second item represents the average throughput from fading variability. Also, (15) reveals an interesting merit of PFS: each user's throughput is solely determined by its own channel statistics when the instantaneous rate is Gaussian.

We would like to point out that our analysis applies to both Rayleigh and Rician fading cases while almost all existing analysis on PFS are only for Rayleigh fading. 
Now we have developed our mathematical tool to analyze the PFS algorithm in SISO/SIMO/MISO/MIMO cellular network, we will extend it to multi-antenna mesh cases.

\section{B. PFS-Extension to DPMAM Networks}

In a DPMAM network, each node can be considered as a BS serving its neighbors within communication range.

Denote $\Omega_{i}$, and $\Phi_{i}$ are node $n_{i}$ 's transmit neighbor set (i.e., nodes in the set can transmit to $n_{i}$ ) and receive neighbor set (i.e., nodes in the set can receive from $n_{i}$ ), respectively. Let $R_{i, j}$ is the feasible data rate between $n_{i}$ and $n_{j}$. One can readily have

$$
\left\{\begin{array}{l}
E\left[\alpha_{j}\right] \approx \sum_{n_{i} \in \Omega_{j}}\left[\frac{E\left[R_{i, j}\right]}{N_{j}+1}+\int_{-M_{i, j}}^{\infty} y \sigma_{R_{i, j}} f_{(0,1)}(y)\left[F_{(0,1)}(y)\right]^{N_{j}} d y\right] . \\
E\left[\beta_{j}\right] \approx \sum_{n_{i} \in \Phi_{j}}\left[\frac{E\left[R_{j, i}\right]}{N_{i}+1}+\int_{-M_{j, i}}^{\infty} y \sigma_{R_{j, i}} f_{(0,1)}(y)\left[F_{(0,1)}(y)\right]^{N_{i}} d y\right]
\end{array}\right.
$$

where $N_{j}$ is the number of neighbors that $n_{j}$ can receive from, $\alpha_{j}$ and $\beta_{j}$ are the incoming and outgoing data rate of $n_{j}$, $M_{i, j}=E\left[R_{i, j}\right] / \sigma_{R i, j}, E\left[R_{i, j}\right]$ and $\sigma_{R i, j}$ are the expect value and standard deviation of $R_{i, j}$ given by (1) and (2) for SIMO case, respectively.

We now conduct simulations to justify our theoretical results for the DPMAM network.

\section{NUMERICAL RESUlTS AND SIMULATION}

We first plot in Figure 2 the comparison of our theoretical expression and the existing analytic result given by (3) in a cellular network for both SISO and MIMO cases. The network has 10 users with average $\operatorname{SINR}=1,2,3, \ldots, 10 \mathrm{~dB}$, respectively.

Comparatively, our theoretical result provides accurate estimation of the PFS throughput for both SISO and MIMO cases while existing analytic expression is valid only for SISO cases (For SISO cases, the theoretical results from both approaches are in fact undistinguishable).

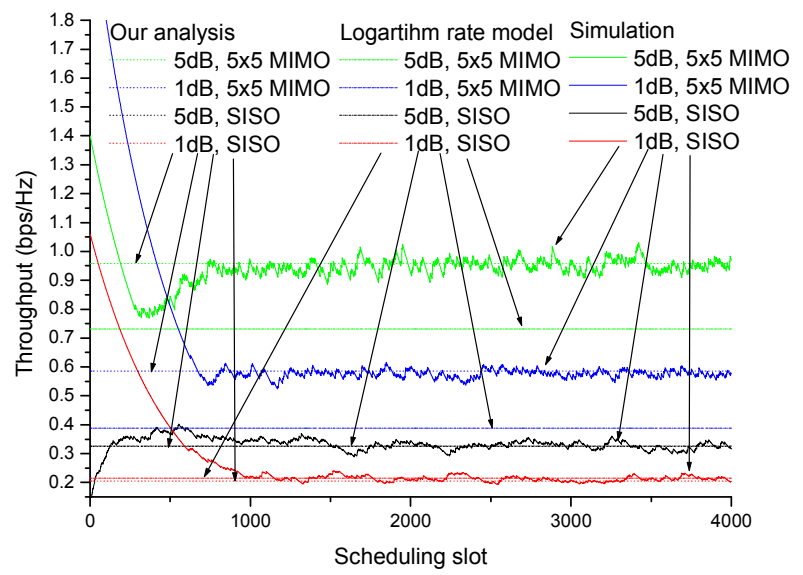

Figure 2. Outgoing and incoming data rate of the 1-dB and 5-dB users

For the same network, we also plot in Figure 3 and 4 the simulation results of $E\left[\mu_{i}\right] / E\left[R_{i}\right]$ and $E\left[\mu_{i}\right] / E\left[\sigma_{i}\right]$ for various user $i$. From (1) and (2), we know that higher average SINR corresponds to higher $E[R]$ and $\sigma_{R}$. Figure 3 and 4 clearly show that, for the PFS over Rayleigh fading channels, one will have $\sigma_{R i} / \sigma_{R j} \leq E\left[\mu_{i}\right] / E\left[\mu_{j}\right] \leq E\left[R_{i}\right] / E\left[R_{j}\right]$ given $0 \leq E\left[R_{j}\right] \leq E\left[R_{i}\right]$.

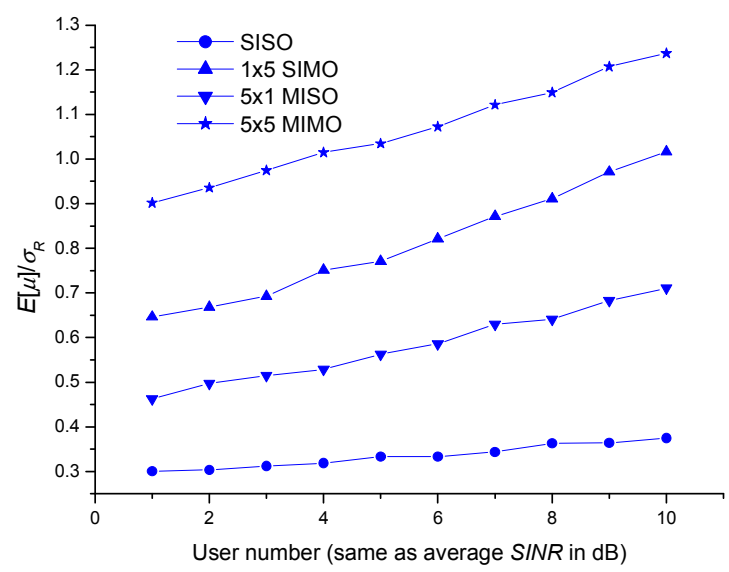

Figure 3. $E[\mu] / E[R]$ in Rayleigh fading, PFS-enable network (10 users)

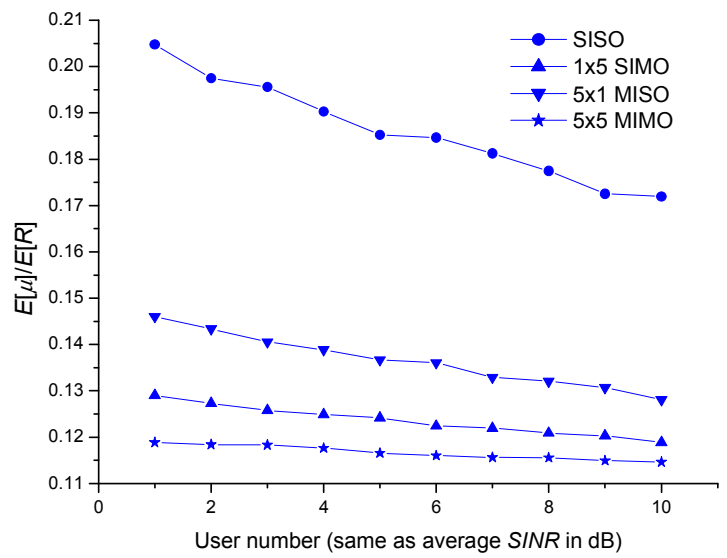

Figure 4. $E[\mu] / E[\sigma]$ in Rayleigh fading, PFS-enable network (10 users)

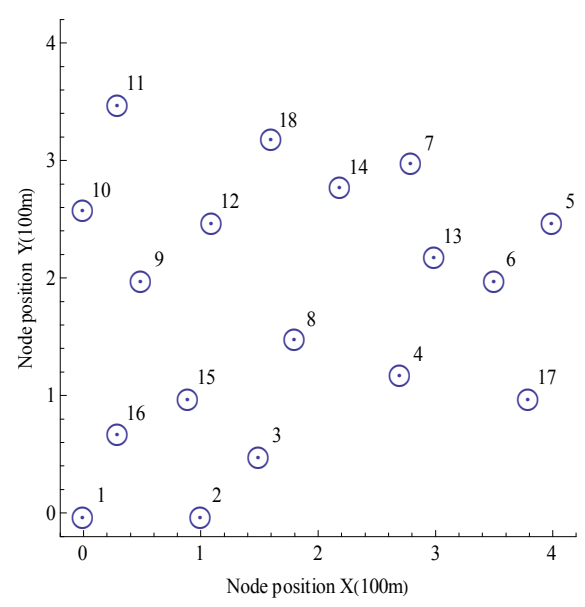

Figure 5. 18-node wireless mesh network

We now use (16) to evaluate our theoretical analysis of PFS for DPMAM networks. Simulation parameters are:

- 4000 scheduling slots, moving average factor $k=500$.

- Each user has independent Rayleigh fading channel. For a $1 \times r$ SIMO link, the received SINR has a probability density function $p d f(\lambda)=\lambda^{n-m} e^{-\lambda} /(r-1)$ ! [8]. 
- In Figure 5, 18 nodes are randomly placed in an area of $420 \mathrm{~m} \times 420 \mathrm{~m}$. Each node has 5 transmit and receive antennas. Neighboring nodes use $1 \times 5$ SIMO for communication. The received average $S I N R$ is given by

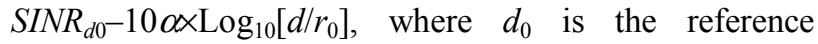
distance which is taken to be $50 \mathrm{~m}, \operatorname{SINR}_{d 0}=20 d B$ is the received SINR at $d_{0}, \alpha$ is the path loss exponent ( $\alpha$ is typically $2 \sim 5), r_{0}=110 \mathrm{~m}$ is the transmit range, and $r$ is the distance between communicating nodes.

The validity of the theoretical analysis for the DPMAM network is illustrated in Figure 6.

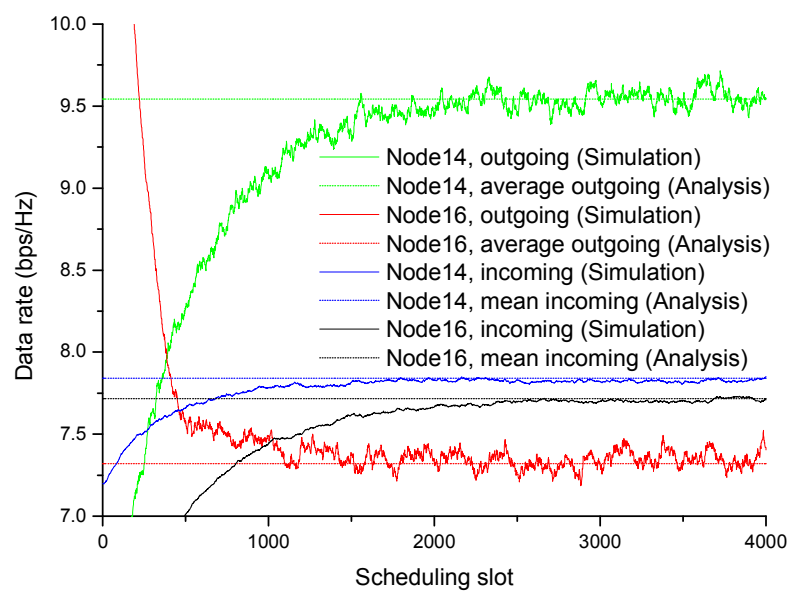

Figure 6. Outgoing and incoming data rate of node-14 and node- 16 .

The nature of relatively large fluctuations in Figure 6 for outgoing data rate lies in the fact that a node could be scheduled by different nodes.

\section{CONCLUSIONS AND FUTURE WORK}

We proposed a full duplex distributed PFS-enabled multiantenna mesh (DPMAM) network. Simulations show that the conventional analytic approach assuming the logarithm rate model is no longer valid for such networks. To study the DPMAM network, a GA-based mathematical framework for the PFS research is developed. The analytic framework presented in the paper has some advantages over the traditional analytic approaches on PFS:

a. It is mathematically graceful;

b. It can be used to analyze the PFS performance in SISO/SIMO/MISO/MIMO environments while the conventional approach is valid only for SISO cases;

c. While existing theoretical results are only for Rayleigh fading, the mathematical framework presented here can be used to analyze the PFS algorithm in both Rayleigh and Rician fading environments.

d. Under the framework, the theoretical results from SISO, cellular networks can be directly extended to the proposed DPMAM network.
The results from the theoretical framework are being integrated into the MEMBRANE project [10].

As the first step into the study of PFS in wireless mesh networks, for the DPMAM network shown in Figure 1, we assume each node has enough transmit antennas and SIMO is used for the communication between two nodes. This assumption could be relaxed when there is cooperation among nodes. For instance, denote $n_{i} \underset{t \times r}{\rightarrow} n_{j}$ the $t \times r \quad$ MIMO communication between $n_{i}$ and $n_{j}$, let say each node has 2 transmit antennas and 2 receive antennas, when $n_{3}$ is $P F$ scheduled by 3 neighbors $\left(n_{1}, n_{2}\right.$ and $\left.n_{6}\right)$ simultaneously, a smart cooperation method would choose a preferred one of the 6 possible patterns according to some criteria: $\left\{\left(n_{3} \underset{1 \times 2}{\rightarrow} n_{1}, n_{3} \underset{1 \times 2}{\longrightarrow} n_{2}\right),\left(n_{3} \underset{1 \times 2}{\rightarrow} n_{1}, n_{3} \underset{1 \times 2}{\rightarrow} n_{6}\right),\left(n_{3} \underset{1 \times 2}{\rightarrow} n_{2}, n_{3} \underset{1 \times 2}{\rightarrow} n_{6}\right),\left(n_{3} \underset{2 \times 2}{\rightarrow} n_{1}\right),\left(n_{3} \underset{2 \times 2}{\longrightarrow} n_{2}\right),\left(n_{3} \underset{2 \times 2}{2 \times 2} n_{6}\right)\right\}$

. Node cooperation in such scenario is an interesting topic and will be one of our future work. Frequency-reuse can also be used to reduce the number of neighbors to eliminate the need for large number of transmit antennas per node. Another research direction is to introduce proportional fairness in allocating subchannels to different nodes.

\section{ACKNOWLEDGMENT}

This research was financially supported by grant from EU IST FP6 MEMBRANE project under the contract 027310.

\section{REFERENCES}

[1] F. Kelly, "Charging and Rate Control for Elastic Traffic", Eur. Trans. on Telecommun., February 1997, pp. 33-37.

[2] A.Jalali, R. Padovani and R. Pankai, "Data Throughput of CDMA-HDR: a High Efficiency-High Data Rate Personal Communication Wireless System," in Proc. Veh. Technol. Conf., January. 2001, pp. 55-67.

[3] J. M. Holtzman, "Asymptotic analysis of proportional fair algorithm," in Proc. IEEE PIMRC, San Diego, CA, 2001, pp. 33-37.

[4] H. J. Kushner and P. A. Whiting, "Asymptotic Properties of Proportional-Fair Sharing Algorithms: Extensions of the Algorithm," in Proc. of the Annual Allerton Conference on Communication, Control and Computing, vol. 41, 2003, pp. 303-311.

[5] S. Borst, "User-level performance of channel-aware scheduling algorithms in wireless data networks," in Proc. INFOCOM, San Francisco, 2003, pp. 321-331.

[6] D. Avidor, S. Mukherjee, J. Ling and C. Papadias, "On Some Properties of the Proportional Fair Scheduling Policy," in Proc. IEEE PIMRC, New Jersey, September 2004, pp. 853-858.

[7] J-G. Choi and S. Bahk, "Cell-Throughput Analysis of the Proportional Fair Scheduler in the Single-Cell Environment," IEEE Trans. Veh. Technol., vol. 56, no. 2, pp. 766-778, March 2007.

[8] I. E. Telatar, "Capacity of multi-antenna Gaussian channels," European Transactions on Telecommunications, Vol.10, No.6, pp.585-595, November/December 1999.

[9] P. J. Smith and M. Shafi, "On a Gaussian Approximation to the Capacity of Wireless MIMO Systems," in Proc. IEEE ICC, New York, April 2002, pp. 406-410.

[10] The MEMBRANE website. [Online]. Available: http://www.imperial.ac.uk/MEMBRAN 